\title{
Research of Information Management System
}

\author{
Xue Xing ${ }^{\text {a }}$, Queyue Zhao \\ Jinlin Institute of Chemical Technology, Jilin 132022, China \\ apatricia_xx@126.com
}

Keywords: Information Management System, JFreeChart, J2EE, Systems Analysis.

\begin{abstract}
Information management system is an important part of enterprise management, and intelligent information processing system for business information collection, collation, processing and utilization has become an inevitable trend. The design from reality, business information management system based on J2EE to achieve the dynamic handling of business information management processes, thereby improving enterprise management level and the quality of decisions.
\end{abstract}

\section{Introduction}

Information management system is helpful to explore potential business for the enterprise, the enterprise operation efficiency fully, continuously expand the market space, keep old customer to attract new customers. The traditional artificial way management, the efficiency is low, the secrecy bad, produce a large number of documents and data, to find, update and maintenance have brought difficulties. An intelligent information processing system avoiding the shortcomings of traditional way of management, implement the customer management flexibility, timeliness. The investment and use of business information management system is not only the customer knowledge management model and the use of data mining technology, the implementation of business information management process is value-oriented and competition ability, help enterprises to realize intelligent decision analysis of the customer, is bound to be large-scale popularization and application.

\section{General Instructions}

Main research. In this paper, according to the enterprise actual demand, in the study of dynamic server page technology, on the basis of system structure and database access technology, using JSP and open source MySQL database engine for development, we design a Business information management system based on J2EE technology. This system in particular is using JFreeChart chart, in the implementation of the system elements (customer information) statistical analysis, and adopted the strict access control and flexibility, the non-recoverable MD5 encryption algorithm, the system has strong flexibility and operability, practicability, security, etc., in order to improve the management level of enterprises and the quality of decision-making is convenient for archive and enterprise customer management [1], avoid due to factors such as personnel changes lead to the risk of loss of customer resource.

The system developed by technical personnel, with a relatively low cost, and customer information management system can be according to computer records the data of [2], constantly adjust production development tendency of the enterprise, win, develop, maintain valuable customers, thus increasing corporate income, optimize profitability. This system based on J2EE MVC (model, view and controller) [3] a three-tiered architecture, using SSH framework (Struts + spring + Hibernate is the more popular a kind of open source Web application framework [4]) to complete the design. 


\section{Demand Analysis}

\subsection{System functional requirements.}

Business model of business information management system (BIMS), which is the generalization of the overall business of the whole system function and description, can help users to grasping the customers using the system. Business information management system function model is shown in figure 1.

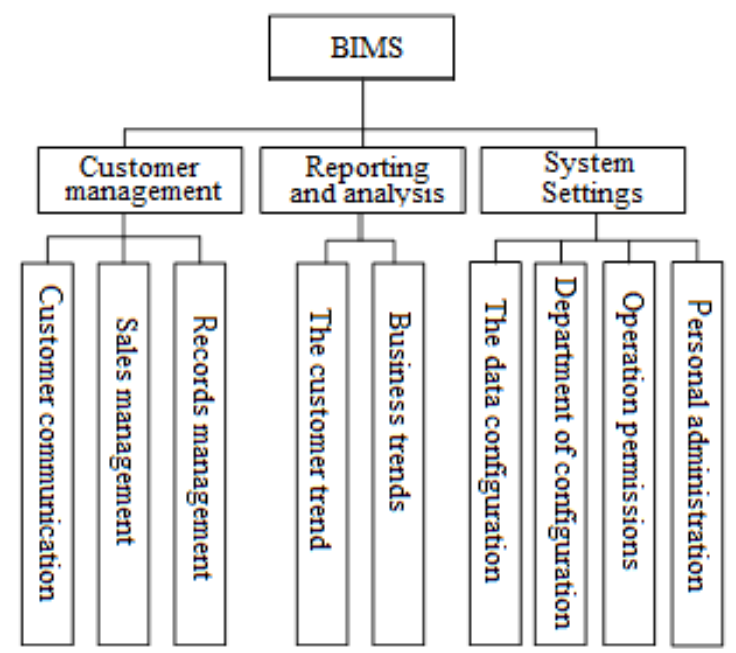

Fig.1 Business information management system function structure

\subsection{System business processes.}

Operation is flexible and fast, for the convenience of enterprise management system to a stable, safe and reliable data storage, data processing and transmission requires security [5], the systematic execution can master both managers and users, flow chart of building is shown in figure 2 .

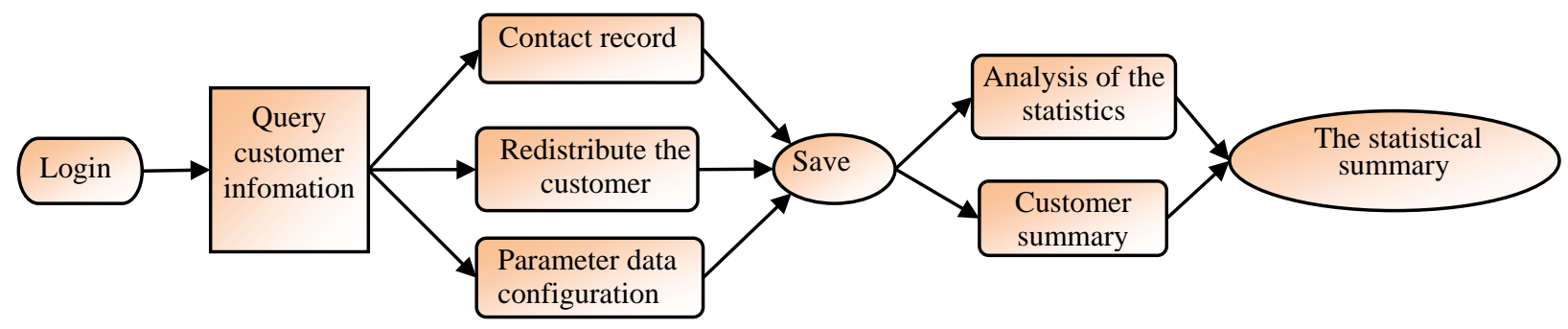

Fig.2 Business flow diagram

\section{System Implementation}

The overall. modularAccording to the practical operation of the business management process, the entire system uses layers of management mode, and the whole function is divided into modules.

Modular design of customer communication. Module implements some of the major attributes according to the customer (customer name, customer level, customer source, nature of the customer, customer pinyin code, telephone) fuzzy query, real-time update the display, etc.Module to achieve the customer data (basic information, enterprise information, other information) of the increase, delete, view, modify, and other functions.Module implements the module sharing operation (e.g., customer data sharing).Module implements the client handlers change operation.Module can realize the function set or change the customer contact time next time.Module implements the customer related contacts, contact record to view, and according to the conditions query related contacts, contact record function.Module implements all clients, need to contact the customer today, expired contact customers, real-time query function under different conditions.

Modular design of sale management. Module implements some of the major attributes according to the salesman (name, gender, position, department, office phone, mobile phone) fuzzy query, 
real-time update the display, etc. Module implements the salesman information (basic information, other information), increase, delete, view, modify, and other functions.

Modular design of record management.Module is realized by some main properties of the contact record (contact, contact category, contact time, the salesman) fuzzy query, real-time update the display, etc.

Module implements the contact record (basic information, other information), increase, delete, view, modify, and other functions.

Chart analysis module. Module implements according to the customers information is related important elements (customer, enterprise, and the company size, and name, customer level) to form the corresponding histogram. Module implements the histogram information clear, clear and updated in real time display, can be intuitive and effective assessment and analysis. Module implements according to the customers information is related important elements (customer, enterprise, the company size, and name, customer level) to form the corresponding reports. Module implements the report information clear, accurate and updated in real time display, can be intuitive and effective assessment and analysis.

Data configuration module. Module, and realized the linkage can be carried out in accordance with the province name query. Module implements the provinces, cities and the increase of information, delete, view, modify, and other functions. Fuzzy query module implements the department name, updated in real time display, etc. Module to achieve the department's increase, delete, view, modify, and other functions, under the department personnel is not allowed to delete. The fuzzy query module implements the permissions group name and updated in real time display, etc. Module implements the privilege group increase, delete, view, modify, and other functions, including permissions set when a user is not allowed to delete. Accessible to a specific module implements the permissions set menu Settings. The specific function of module implements the permissions set of operational Settings.

Modular design of personal administration. Module implements the user some important attributes (user name, Chinese name, department, user status) fuzzy query, real-time update the display, etc. Module achieve the user information (basic information, detailed information, professional skills, information system) of add, view, and modify, enabled, disabled, and other functions. Module implements the user password changes.

\section{All Levels Function Analysis and Design}

\subsection{Modular design of executives of Company management and hierarchical management department.}

Salesman functional allocation management mainly for different users to assign different permissions, and its corresponding function, including the users increase, delete, view, modify, agent distribution rights, salesman, basic information and password management core distribution function is operating group permissions Settings, the main rights group name of fuzzy query, real-time update the display. Implement permissions set when a user is not allowed to delete. Achieve privilege group can access the specific menu Settings. Implement the specific function of permissions set of operational Settings. 


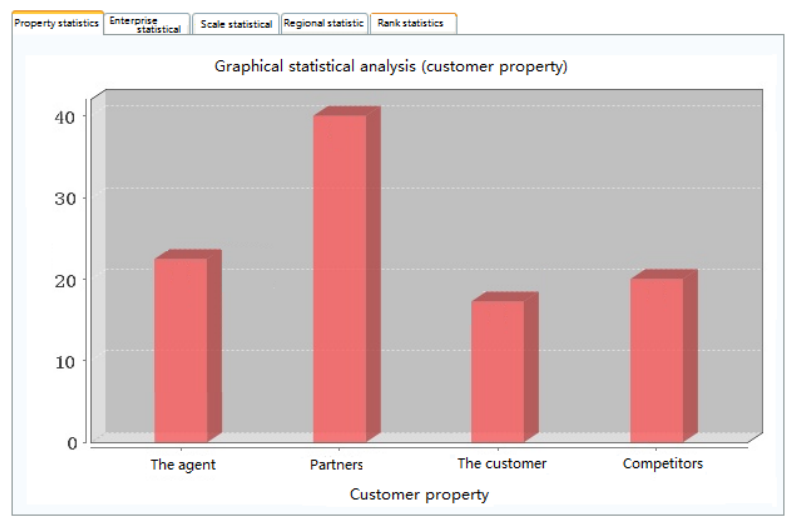

Fig.3 Statistical analysis graphical interface

Customer resource chart analysis module is mainly to the main elements in the client data sorting analysis, which is divided into two forms. The first is a chart analysis, realizes the customer data of related important elements (customer, enterprise, the company size, name, customer level) as the abscissa, the number of generated histogram for the ordinate, and the histogram information clear, clear and updated in real time display, can be intuitive and effective assessment and analysis, statistical analysis graphics page is shown in figure 3. The second is the customer classification statistics, project implementation can see to classification (customer, enterprise, the company size, name, customer level) and the customer number for the grouping of the corresponding statistical reports, and report the information clear, accurate and updated in real time display can be intuitive and effective assessment and analysis, customer classification statistics.

\subsection{Modular design of the classification management departments.}

Hierarchical management departments and each department clerk, the function is mainly to achieve customer visit record management, business contact management and personnel management.

The customer visit record management module is mainly to achieve some of the major attributes according to the customer (customer name, customer level, customer source, customer property, etc.) for fuzzy query, real-time update the display, and client data add, delete, view, modify, and other functions. Realization of the sharing operation module, contact customer handlers changes, changes the next time, etc. Implementation related to customer contact, contact record to view, and according to the conditions query related contacts, contact record function. Implement all clients, need to contact the customer today, not expired contact customers real-time query functions. Business personnel management module and contact records management module is mainly to achieve the basic information add, delete, view, modify, and other functions.

\subsection{Database design.}

In system design, database is the base of data management, it stores the system all the data and the data connection. Development database in the design of Business information management system main entities are customers, the salesman, contact record. The main business of the customer relationship of the whole system is carried out around the three entities.

\section{Web Application System Test}

The security of Web application system from the point of use can be divided into application-level security and transport level security. This paper mainly related to the application level of security problems, the main purpose of application-level security testing is to find a Web system itself security hidden danger existing in program design, test area can be divided into using registered before landing the way into the page. The Web application system is set a time when the login control. The $\log$ file is a powerful guarantee for the safety of the Web application system. General Web application system uses secure sockets. 


\section{Summary}

Overall design identified in B/S mode, on the JAVA platform to the layered design of system, and the system to take certain safety precautions, including user login password using MD5 encryption algorithm, landing interface set up verification code verification, mainly to prevent illegal users access the database or use illegal means to malicious tampering with data. Design diagram development technology, realizing the the management, customer management, report and analysis system, and other functions. From the actual operation effect of the system, can satisfy the requirement of the basic processing, help enterprise rapid and timely grasp the customer information and market situation, improve the efficiency of enterprises.

\section{References}

[1] J.F. Zhao: Journal of the Northern Industrial University, Vol. 22 (2010) No.2, p.11.

[2] L. Chen.: Journal of Management Engineering, Vol. 20 (2006) No.4, p.41.

[3] L. Zhang: Computer Engineering, Vol. 21 (2011), p.255.

[4] S.Z. Li, N.P. Zhang, G.P. Chang: Computer Engineering, Vol. 22 (2006), p.274

[5] Z.H. Su: Establish and Maintain Customer Relationship, China 2007.

[6] R.X. Lu, Z. Yu, Y.L. Ruan: Computer Application Research, Vol. 20 (2003) No.3, p.140. 